\title{
Weak Zero-Knowledge Beyond the Black-Box Barrier
}

\author{
Nir Bitansky \\ nirbitan@tau.ac.il \\ Tel Aviv University \\ Tel Aviv, Israel
}

\author{
Dakshita Khurana \\ dakshkhurana@gmail.com \\ Microsoft Research \\ Cambridge, Massachusetts, USA \\ University of Illinois \\ Urbana-Champaign, Illinois, USA
}

\author{
Omer Paneth \\ omerpa@mit.edu \\ MIT \\ Cambridge, Massachusetts, USA
}

\begin{abstract}
The round complexity of zero-knowledge protocols is a longstanding open question, yet to be settled under standard assumptions. So far, the question has appeared equally challenging for relaxations such as weak zero-knowledge and witness hiding. Protocols satisfying these relaxed notions under standard assumptions have at least four messages, just like full-fledged zero-knowledge. The difficulty in improving round complexity stems from a fundamental barrier: none of these notions can be achieved in three messages via reductions (or simulators) that treat the verifier as a black box.

We introduce a new non-black-box technique and use it to obtain the first protocols that cross this barrier under standard assumptions. We obtain weak zero-knowledge for NP in two messages, assuming the existence of quasipolynomially-secure fullyhomomorphic encryption and other standard primitives (known based on the quasipolynomial hardness of Learning with Errors), and subexponentially-secure one-way functions. We also obtain weak zero-knowledge for NP in three messages under standard polynomial assumptions (following for example from fully homomorphic encryption and factoring). We also give, under polynomial assumptions, a two-message witness-hiding protocol for any language $\mathcal{L} \in \mathbf{N P}$ that has a witness encryption scheme. This protocol is publicly verifiable.

Our technique is based on a new homomorphic trapdoor paradigm, which can be seen as a non-black-box analog of the classic FeigeLapidot-Shamir trapdoor paradigm.
\end{abstract}

\section{CCS CONCEPTS}

- Theory of computation $\rightarrow$ Cryptographic protocols; $\bullet$ Security and privacy $\rightarrow$ Mathematical foundations of cryptography.

\section{KEYWORDS}

homomorphic trapdoor, zero-knowledge, non black-box simulation, witness hiding

Permission to make digital or hard copies of all or part of this work for personal or classroom use is granted without fee provided that copies are not made or distributed for profit or commercial advantage and that copies bear this notice and the full citation on the first page. Copyrights for components of this work owned by others than ACM must be honored. Abstracting with credit is permitted. To copy otherwise, or republish, to post on servers or to redistribute to lists, requires prior specific permission and/or a fee. Request permissions from permissions@acm.org.

STOC '19, June 23-26, 2019, Phoenix, AZ, USA

(c) 2019 Association for Computing Machinery.

ACM ISBN 978-1-4503-6705-9/19/06 _\$15.00

https://doi.org/10.1145/3313276.3316382

\section{ACM Reference Format:}

Nir Bitansky, Dakshita Khurana, and Omer Paneth. 2019. Weak ZeroKnowledge Beyond the Black-Box Barrier. In Proceedings of the 51st Annual ACM SIGACT Symposium on the Theory of Computing (STOC '19), June 23-26, 2019, Phoenix, AZ, USA. ACM, New York, NY, USA, 12 pages. https://doi.org/10.1145/3313276.3316382

\section{INTRODUCTION}

Zero-knowledge protocols are spectacular. They allow to prove any NP statement without revealing anything but the statement's validity. That is, whatever a malicious verifier learns from the protocol can be efficiently simulated from the statement alone, without ever interacting with the prover. Since their invention [43] and construction for all of NP [41], zero-knowledge protocols have had a profound impact on modern cryptography.

A central question in the study of zero-knowledge is that of round complexity. Zero-knowledge arguments with a negligible soundness error can be achieved in four messages [34], under the minimal assumption of one-way functions [8]. ${ }^{1}$ In terms of lower bounds, zero-knowledge arguments for languages outside BPP, and without any trusted setup, require at least three messages [42].

Zero knowledge protocols with an optimal number of messages (and a negligible soundness error) have been pursued over the last three decades and have proven difficult to construct. Three-message zero-knowledge arguments were only constructed under auxiliaryinput knowledge assumptions, which are considered implausible $[9,12,13,21,48]$, and more recently, under a new, non-standard, assumption on the multi-collision resistance of keyless hash functions [14]. Three-message protocols based on standard cryptographic assumptions remain out of reach.

Relaxing zero-knowledge. Given the current state of affairs, it is natural to consider relaxations of the zero-knowledge privacy guarantee. Three main relaxations considered in the literature are:

- Witness indistinguishability [34]: Ensures that a malicious verifier cannot distinguish between proofs that are generated using different witnesses. While witness indistinguishability is natural and often useful as a building block in applications, it is still quite limited. For example, in the rather common scenario where statements have a unique witness, witness indistinguishability becomes meaningless.

- Witness hiding [34]: Ensures that a malicious verifier cannot learn an entire witness from the proof; that is, unless such a witness can be efficiently computed from the statement alone. In contrast to witness indistinguishability, the

${ }^{1}$ Recall that a protocol is an argument if it is only computationally sound, and a proof if it is statistically sound. 
witness hiding requirement is also meaningful in the unique witness case.

- Weak zero-knowledge [32]: Relaxes zero-knowledge by switching the order of quantifiers. Full-fledged zeroknowledge requires that for every verifier there exists a simulator that generates a view, indistinguishable from the verifier's view in a real interaction, for every distinguisher. In contrast, weak zero-knowledge requires that for every verifier and distinguisher, there exists a simulator that fools this specific distinguisher. We also allow the simulator to depend on the desired distinguishing gap. ${ }^{2}$

Weak zero-knowledge hides any predicate of the statement and witness (used by the prover) that cannot be computed from the statement alone. It implies both witness hiding and witness indistinguishability.

The above relaxations are not subject to the same lower bounds as full-fledged zero-knowledge. In fact, the only known unconditional lower bound rules out weak zero-knowledge in one message [42].

As for constructions, witness indistinguishability has indeed been obtained, under standard assumptions, in three [34, 41], two $[4,31,53]$, and eventually even one message $[6,47]$. In contrast, weak zero-knowledge and witness hiding have proven to be just as challenging to construct as full-fledged zero-knowledge. So far, constructions with less than four messages are known only based on non-standard assumptions, which by now are considered implausible [10, 16, 24], for restricted classes of adversarial verifiers [13, 53], or for restricted classes of languages [34, 57]. (See the related work section for more details).

The black-box barrier. The difficulty in obtaining round-optimal zero-knowledge and its relaxations stems from a fundamental barrier known as the black-box barrier - three-message zeroknowledge is impossible as long as the simulator is oblivious of the verifier's code, treating it as a black box [40]. Similar barriers hold for both weak zero-knowledge and witness hiding [50].

Whereas classical zero-knowledge protocols all have black-box simulators, starting from the breakthrough work of Barak [5], nonblack-box techniques that exploit the verifier's code have been introduced (c.f., [11, 17, 26, 28, 30, 46]). However, existing techniques seem to require at least four messages (except for [14], based on a non-standard assumption).

In conclusion, as in the case of zero-knowledge, weak-zeroknowledge and witness-hiding protocols in three-message or less, based on standard cryptographic assumptions, remain out of reach.

\subsection{Results}

We devise a new non-black-box technique and apply it to obtain, under standard assumptions, weak zero-knowledge and witness hiding beyond the black-box barrier.

Our main result is a two-message weak zero-knowledge argument for NP.

\footnotetext{
${ }^{2}$ There are several variants of this definition strengthening/weakening different aspects $[27,32]$.
}

THEOREM 1.1 (INFORMAL). There exists a two-message weak zeroknowledge argument for NP assuming: subexponentially-secure oneway functions and quasi-polynomially-secure fully-homomorphic encryption, random-self-reducible encryption, two-message witnessindistinguishable arguments and oblivious transfer, non-interactive commitments, and compute-and-compare obfuscation.

All of the above primitives (but subexponentially-secure oneway functions) are known under quasipolynomial hardness of LWE, with the exception of fully-homomorphic encryption that also requires a circular security assumption [4, 22, 23, 39, 44, 45, 53, 59]. We can also replace compute-and-compare obfuscation with fullyhomomorphic encryption scheme with some additional natural properties that are satisfied by known constructions (see the technical overview for more details).

It is interesting to note that the result gives an example of a natural weak-zero-knowledge protocol that is provably not zeroknowledge. Previously, a contrived separation was known assuming exponentially-hard injective one-way functions [27].

Our second result is a three-message protocol that is based only on polynomial hardness assumptions.

Theorem 1.2 (Informal). Assuming polynomial hardness of the primitives in Theorem 1.1, as well as dense commitments, there exists a three-message weak zero-knowledge argument for $\mathbf{N P}$.

The polynomially-hard version of the required primitives (including dense commitments) can be based on (polynomially-hard) fully-homomorphic encryption, LWE, and either Factoring or standard Bilinear-Group assumptions.

Our third result, also from polynomial hardness assumptions, is a two-message witness-hiding protocol for any language $\mathcal{L} \in$ NP that has a witness encryption scheme [37].

Theorem 1.3 (INFORMAL). There exists a two-message witnesshiding argument for any language $\mathcal{L} \in \mathbf{N P}$ under the same (polynomial) assumptions as in Theorem 1.2 and witness encryption for $\mathcal{L}$.

For the time being, witness encryption for all of NP is only known based on indistinguishability obfuscation, or based on nonstandard assumptions on multilinear maps [25, 36]. Witness encryption for several non-trivial languages follows from results on hash proofs systems [29].

The protocol we obtain is publicly verifiable, meaning that the proof can be verified given the transcript alone, without secret verifier randomness. We observe that the [42] lower bound for twomessage zero-knowledge extends also to two-message publiclyverifiable weak zero-knowledge, and thus we cannot expect to get a similar result for weak zero-knowledge.

From explainable to malicious security. The main component in all of the above results is a weak-zero-knowledge argument for NP against a new class of verifiers that we call explainable. Such verifiers may not follow the honest verifier strategy, but they do choose their messages from the support of the honest verifier message distribution; namely, there exist honest verifier coins that explain their behavior. The notion resembles that of semi-malicious and defensible adversaries [20,49], but differs in the fact that the 
verifier does not explicitly choose a random tape for the honest verifier (and it may not be possible to efficiently extract such a tape from the verifier).

THEOREM 1.4 (INFORMAL). Under the same assumptions as in Theorem 1.1 (respectively, 1.2), there exists a two-message (respectively, three-message) weak zero-knowledge argument for NP against explainable verifiers.

We then give general compilers to boost explainable security to malicious security. These compilers may be of independent interest. For instance, they imply that to obtain full-fledged zero-knowledge in three messages, it suffices to consider explainable verifiers.

\subsection{Technical Overview}

We now give an overview of our techniques. We focus on twomessage protocols against explainable verifiers, which is the technical core behind our results. We also explain how to avoid superpolynomial hardness assumptions, at the account of adding one message to this protocol. We then describe the main ideas behind our compilers to malicious security.

Warm up: a witness-hiding protocol. Toward constructing a twomessage weak-zero-knowledge protocol against explainable verifiers, let us first consider the easier goal of witness hiding. Recall that a protocol is witness hiding if there exists a reduction that given as input an NP statement $x$, and the code of a witness-finding verifier, outputs a witness. By a witness-finding verifier, we mean a verifier that given a proof that $x$ is true, finds a witness for $x$ with noticeable probability.

Our protocol follows a classic paradigm by Feige, Lapidot, and Shamir [33]. The first verifier message fixes a so-called trapdoor statement $\tau$. In parallel, the prover and verifier execute a twomessage witness-indistinguishable argument that either the statement $x$ or the trapdoor statement $\tau$ hold. (Throughout the rest of the introduction we ignore the first message of the witnessindistinguishable argument.)

The trapdoor statement $\tau$ is meant to have two properties:

- To a malicious prover, trying to convince the verifier of a false statement $x, \tau$ should be computationally indistinguishable from a false statement. Thus, by the soundness of the witnessindistinguishable argument, the prover should fail.

- A reduction that has the code of an explainable witness-finding verifier should be able to obtain a witness $\rho$ for the trapdoor statement $\tau$. Once such a witness is found, the reduction can use it to generate the witness-indistinguishable argument. By witness indistinguishability, the reduction's proof is indistinguishable from the honestly generated proof and, therefore, the verifier will output a valid witness $w$ for the statement $x$ with noticeable probability.

The main challenge in realizing the above paradigm is to extract the trapdoor witness $\rho$ from the verifier's code. The basic idea behind our non-black-box technique, and what enables such extraction, is what we call the homomorphic trapdoor paradigm.

In our protocol, on top of the trapdoor statement $\tau$, the verifier will send an encryption ct of the witness $\rho$ attesting that $\tau$ holds, using a fully-homomorphic encryption scheme. On one hand, by the security of the encryption scheme, this does not compromise soundness. On the other hand, a reduction that has the code of the witness-finding verifier can obtain a witness $w$ for $x$ under the encryption. To do so, the reduction homomorphically invokes the strategy described before - under the encryption ct, it uses $\rho$ to compute the witness-indistinguishable argument, and obtain the witness $w$ from the verifier.

The above step does not find a witness $w$ in the clear (nor does it extract the trapdoor $\rho$ ). We observe, however, that an encryption of a witness $w$ is already a non-trivial piece of information that could only be obtained when $x$ is a true statement; in fact, we can use it as another trapdoor witness. Concretely, we extend our protocol to include yet another, so-called homomorphic, trapdoor statement $\tau_{h}$ where a witness $\rho_{h}$ for $\tau_{h}$ could be any encryption of a witness $w$ for $x$. That is, $\tau_{h}$ is true if and only if $x$ is true, and a witness $\rho_{h}$ for $\tau_{h}$ is an encryption of a witness $w$ for $x$.

In the extended protocol, the prover gives a witness- indistinguishable argument that either the statement $x$, the trapdoor statement $\tau$, or the homomorphic trapdoor statement $\tau_{h}$ hold. The reduction first uses the encrypted trapdoor $\rho$ homomorphically to obtain a trapdoor $\rho_{h}$ (in the clear), and then uses $\rho_{h}$ to generate the witness-indistinguishable argument. By witness indistinguishability, the verifier will output a witness $w$, this time in the clear.

One difficulty in realizing the above strategy is to prove the homomorphic trapdoor statement $\tau_{h}$; that is, to prove that there exists an encryption $\rho_{h}$ of a valid witness $w$ for $x$, when the reduction lacks the homomorphic decryption key. We discuss how to resolve this difficulty below when describing the more general weak-zero-knowledge protocol.

Toward weak zero-knowledge. Recall that in weak zeroknowledge, we require that there exists a simulator that given the code of a verifier and a distinguisher $D$, simulates the verifier's output so that it fools D. That is, D cannot $\varepsilon$-distinguish between the simulated output and the verifier's output in a real interaction with the prover, for any accuracy parameter $\varepsilon$, where the simulator is allowed to run in time polynomial in $1 / \varepsilon$.

In this setting, the verifier's output is arbitrary and may not include a witness. Thus, we cannot employ the same strategy as before. Nevertheless, our protocol still builds on the homomorphic trapdoor paradigm, but with additional ideas. In a nutshell, instead of extracting a witness $w$ from the verifier under the encryption, we extract a different trapdoor witness from the distinguisher D, under the encryption. Then, as before, we use the encryption of this trapdoor as the homomorphic trapdoor.

Random self-reducible encryption. To enable extraction from the distinguisher, we rely on a public-key encryption scheme that is random self-reducible [19]. In such a scheme, any distinguisher D that can tell encryptions of zero from encryptions of one with advantage $\varepsilon$, under some specific public key pk, can be used to decrypt arbitrary ciphertexts under the key pk, in time polynomial in $|\mathrm{D}| / \varepsilon$. Such schemes are known based on various standard assumptions (such as LWE).

The protocol. We now describe the protocol, and then go on to analyze it.

- The verifier's message, as before, includes a trapdoor statement $\tau$ and a fully-homomorphic encryption ct $=$ 
FHE.Enc sk $(\rho)$ of the corresponding witness $\rho$. In addition, it includes another trapdoor statement $\tau^{\prime}$, and a random self-reducible encryption $\mathrm{ct}^{\prime}=\mathrm{RSR}^{\mathrm{Enc}} \mathrm{pk}_{\mathrm{pk}}\left(\rho^{\prime}\right)$ of the corresponding witness $\rho^{\prime}$. The trapdoor statements $\tau, \tau^{\prime}$ are both indistinguishable from false statements. The statements $\tau, \tau^{\prime}$ also fix a homomorphic trapdoor statement $\tau_{h}$, asserting that "there exists a fully-homomorphic encryption $\rho_{h}$ of a valid witness $\rho^{\prime}$ for $\tau^{\prime}$." That is, $\tau_{h}$ is true if and only if $\tau^{\prime}$ is true, and any witness $\rho_{h}$ for $\tau_{h}$ is a fully-homomorphic encryption of a witness $\rho^{\prime}$ for $\tau^{\prime}$.

- The prover, as before, gives a witness-indistinguishable argument, but now, in addition, it also sends a randomself-reducible encryption of one $\operatorname{ctp}_{\mathrm{p}}=\operatorname{RSR}_{\mathrm{Enc}} \mathrm{Ek}_{\mathrm{p}}(1)$. The witness-indistinguishable argument attests that either one of the statements $x, \tau, \tau_{h}$ hold, or ctp is an encryption of zero (and not one). Note that the trapdoor statement $\tau^{\prime}$ is not directly involved in the witness-indistinguishable argument, but only defines the homomorphic trapdoor statement $\tau_{h}$.

- The verifier checks that the witness-indistinguishable argument is valid and that ctp decrypts to one.

Soundness. Relying on the security of both encryption schemes, we would like to argue that the verifier's encryptions of $\rho$ and $\rho^{\prime}$ can be changed to encryptions of garbage. Then, the trapdoor statements $\tau$ and $\tau^{\prime}$ can be changed to false statements, in which case the homomorphic trapdoor statement $\tau_{h}$ also becomes false. Also, $c t_{p}$ must not be an encryption of zero or the verifier rejects. Soundness then follows from that of the witness-indistinguishable argument.

One subtlety in the above argument is that the verifier's decision depends on the secret key of the the random-self-reducible encryption, and thus changing the encryption of $\rho^{\prime}$ to garbage may affect the bit underlying the prover's encryption ct $_{p}$ and accordingly also the verifier's decision bit. To get around this, we require that the prover exhibits that it "knows" the contents of the encryption ctp (and therefore does not maul the encryption of $\rho^{\prime}$ ). In this case, we can argue that the verifier continues to accept, even if we change the encryption of $\rho^{\prime}$. To facilitate such a proof of knowledge in two messages we resort to complexity leveraging, which is the cause of reliance on super-polynomial assumptions in our two-message protocol. In the three-message setting, we rely instead on extractable commitments based on polynomial hardness assumptions.

Weak zero-knowledge. To argue weak zero-knowledge, we follow a similar approach to that taken in previous works that constructed weak zero-knowledge $[16,53]$. The simulation strategy will have two modes: a secret mode and a public mode, with two corresponding distributions on proofs, $\Pi_{\mathrm{s}}$ and $\Pi_{\mathrm{p}}$. The secret distribution $\Pi_{\mathrm{s}}$ is always indistinguishable from the real distribution $\Pi$ generated by the honest prover, but sampling from this distribution requires a secret s. The public distribution $\Pi_{p}$ can be publicly sampled without knowing s. While $\Pi_{p}$ is not indistinguishable from $\Pi$, to tell them apart, the distinguisher must "know" the secret s. That is, given any distinguisher $D$ that $\varepsilon$-distinguishes $\Pi_{p}$ from $\Pi$, it is possible to extract the secret $s$ in time polynomial in $|D| / \varepsilon$.

This gives rise to a simple simulation strategy that treats separately two types of distinguishers: those that know the secret s, and those that do not. Specifically, given the code of the distinguisher
$D$ and the required simulation accuracy $\varepsilon$, first try to extract the secret $s$ from $D$, and if successful, sample from $\Pi_{s}$ to simulate the proof. Otherwise, deduce that D cannot $\varepsilon$-distinguish $\Pi_{p}$ from $\Pi$, and sample the proof from $\Pi_{p}$. As before, the main challenge in realizing this strategy is to extract the secret $\mathrm{s}$ from $\mathrm{D}$. Our solution again relies on the homomorphic trapdoor paradigm.

Going back to our protocol, let us define the corresponding secret and public distributions $\Pi_{\mathrm{s}}, \Pi_{\mathrm{p}}$ :

- The secret distribution $\Pi_{\mathrm{S}}$ is associated with the homomorphic trapdoor $\tau_{h}$, and can be sampled using any witness $\rho_{h}$ for $\tau_{h}$. Like the real proof distribution $\Pi$, it consists of an encryption of one ctp $=$ RSR.Enc $\mathrm{pk}_{\mathrm{pk}}(1)$. However, differently from the real proof distribution, the witnessindistinguishable argument is computed using the homomorphic trapdoor witness $\rho_{h}$.

- The public distribution $\Pi_{p}$ consists of an encryption of zero $\mathrm{ct}_{\mathrm{p}}=\mathrm{RSR} \cdot \mathrm{Enc}_{\mathrm{pk}}(0)$, and the witness- indistinguishable argument is computed using the randomness of the encryption ctp as a witness.

We argue that $\Pi_{s}$ and $\Pi_{p}$ have the required properties. The fact that $\Pi_{s}$ is indistinguishable from the real proof distribution $\Pi$ follows from witness indistinguishability. We now show that any distinguisher D between $\Pi_{\mathrm{p}}$ and $\Pi$ can be used to extract a witness $\rho_{h}$ (namely, an encryption of a witness $\rho^{\prime}$ for $\tau^{\prime}$ ), which in turn, can be used for sampling from $\Pi_{\mathrm{s}}$. We do this in two steps:

(1) We show that given a distinguisher $D$ between $\Pi_{p}$ and $\Pi$, as well as the trapdoor $\rho$, we can obtain a distinguisher $\mathrm{D}^{\prime}$ that can tell apart encryptions of one from encryptions of zero (with about the same advantage). By random self-reducibility, such a distinguisher $\mathrm{D}^{\prime}$ can be used to decrypt arbitrary ciphertexts under the random self-reducible scheme. In particular, such $D^{\prime}$ can be used to decrypt the encryption $\mathrm{ct}^{\prime}$ of the trapdoor $\rho^{\prime}$ (given in the first verifier message).

The distinguisher $\mathrm{D}^{\prime}$ is defined in the natural way: given a bit encryption, it samples on its own a witness-indistinguishable argument, using $\rho$ as the witness, and then applies D. By witness indistinguishability, the induced distribution $\Pi_{0}$, corresponding to encryptions of zero, is indistinguishable from the real proof distribution $\Pi$. Similarly, the distribution $\Pi_{1}$, corresponding to encryptions of one, is indistinguishable from the public distribution $\Pi_{p}$. Accordingly, $D^{\prime}$ has roughly the same advantage as D.

(2) In the second step, we extract the required trapdoor $\rho_{h}$ allowing us to sample from $\Pi_{s}$. Analogously to the witness-hiding reduction we have already seen, this is done by homomorphically applying the first step - under the encryption ct sent by the verifier, we use $\rho$ to obtain the distinguisher $\mathrm{D}^{\prime}$ and decrypt $\mathrm{ct}^{\prime}$. This results in the required trapdoor $\rho_{h}$, a fully-homomorphic encryption of the trapdoor $\rho^{\prime}$.

How to prove homomorphic trapdoor statements. To conclude our sketch of the weak zero-knowledge analysis, we explain how to prove the homomorphic trapdoor statement $\tau_{h}$. As already mentioned, the difficulty in proving that there exists an encryption $\rho_{h}$ of a valid witness for $\tau^{\prime}$ is that the simulator does not have the corresponding secret key. Next, we discuss two possible solutions. 
The first approach (which we follow in the body of the paper) is based on obfuscation for compute and compare programs. A compute and compare program $\mathrm{CC}[f, u]$ is given by a function $f$ (represented as a circuit) and a target output string $u$ in its range; it accepts every input $x$ such that $f(x)=u$, and rejects all other inputs. A corresponding obfuscator compiles any such program into a program $\widetilde{\mathrm{CC}}$ with the same functionality. In terms of security, provided that the target $u$ has high entropy, the obfuscated program is computationally indistinguishable from a simulated program that rejects all inputs. Such obfuscators are defined and constructed under LWE in $[45,59] .^{3}$

Using compute-and-compare obfuscation, we modify our protocol as follows. We no longer sample a trapdoor statement $\tau^{\prime}$, but instead, set $\rho^{\prime}$ to be a random string. The homomorphic trapdoor statement $\tau_{h}$ is still defined so that a witness $\rho_{h}$ is a fully-homomorphic encryption of $\rho^{\prime}$. Specifically, $\tau_{h}$ is given by an obfuscation $\widetilde{C C}$ of the program CC $\left[\mathrm{FHE} . \mathrm{Dec}_{\mathrm{sk}}, \rho^{\prime}\right]$ that accepts fully-homomorphic ciphertexts that decrypt to $\rho^{\prime}$. Accordingly, a ciphertext $\rho_{h}$ is a valid witness for $\tau_{h}$ if and only if $\widetilde{\mathrm{CC}}\left(\rho_{h}\right)=1$. The obfuscation $\widetilde{\mathrm{CC}}$ will now be specified as part of the verifier's first message, which also includes as before the trapdoor statement $\tau$, a fully-homomorphic encryption of the trapdoor witness $\rho$, and a random self-reducible encryption of the random string $\rho^{\prime}$.

The simulator can obtain a fully-homomorphic encryption of $\rho^{\prime}$ and use it as a trapdoor witness $\rho_{h}$. Furthermore, as required for soundness, $\tau_{h}$ is indistinguishable from a false statement, since $\widetilde{\mathrm{CC}}$ is indistinguishable from a program that rejects all inputs.

Another approach, without compute-and-compare obfuscation. We now describe an alternative approach for proving the trapdoor statement, which does not rely on compute-and-compare obfuscation, but requires the homomorphic encryption to satisfy additional properties.

Here, given the fully-homomorphic encryption $\rho_{h}$ of $\rho^{\prime}$, the simulator homomorphically evaluates the NP witness verification procedure for the statement $\tau^{\prime}$ and sends the encrypted output bit. It then proves that this bit encryption was indeed obtained by homomorphically evaluating the verification procedure for $\tau^{\prime}$ using the encryption $\rho_{h}$ as a witness. The verifier checks the proof and in addition decrypts the output bit and checks that it is accepting.

There are some subtleties to take care of: a) to preserve witness indistinguishability, the homomorphic evaluation must be function hiding and b) to preserve soundness, the prover must convince the verifier that the homomorphic computation was performed over a valid ciphertext $\rho_{h}$. To this end, we require a validation operation mapping arbitrary (possibly invalid) ciphertexts into valid ones, while preserving the plaintext underlying valid ciphertexts. Both properties can be achieved in existing fully-homomorphic encryption constructions (without additional assumptions) [38, 52, 56]. (Also, a similar malleability problem as described before also occurs here and is dealt with using a proof of knowledge.)

Another issue is that the simulator cannot tell whether the encryption $\rho_{h}$ it obtained is indeed an encryption of a valid trapdoor witness $\rho^{\prime}$ or not (in which case, it should deduce that D cannot tell $\Pi$ from $\Pi_{p}$ and use $\Pi_{p}$ to simulate). To deal with this, we again

\footnotetext{
${ }^{3}$ The known construction have a one-sided negligible correctness error. This error will not obstruct our protocol and is ignored in this introduction.
}

use the distinguisher $\mathrm{D}^{\prime}$ to decrypt $\mathrm{ct}^{\prime}$, except that we do so in the clear. Since now we do not have the trapdoor witness $\rho$, we use $\rho_{h}$ instead. By witness indistinguishability, if we fail to decrypt $\mathrm{ct}^{\prime}$ and obtain the witness $\rho^{\prime}$ in the clear, we can deduce that D cannot tell $\Pi$ from $\Pi_{\mathrm{p}}$.

1.2.1 From Explainable to Malicious. Observe that in the protocols described above, it was crucial that the verifier behaves in an explainable fashion. In particular, the simulation strongly relies on the fact that the verifier's fully-homomorphic encryption ct is indeed an encryption of trapdoor witness $\rho$ for the statement $\tau$. To deal with malicious adversaries, we design compilers that take protocols secure against explainable verifiers and turn them into protocols secure against malicious verifiers. We provide three different compilers for different settings. We now explain the main ideas behind each of these compilers.

A two-message compiler based on super-polynomial assumptions. Our first compiler is based on two-message conditional disclosure of secrets schemes for NP, which is known under standard assumptions $[2,3,16]$. In such a scheme, the verifier first sends an instance $x^{\prime}$ for some NP language $\mathcal{L}^{\prime}$ together with an encrypted witness. The prover responds with an encryption of a message, which the honest verifier can then decrypt. In contrast, if a cheating verifier sends $x^{\prime} \notin \mathcal{L}^{\prime}$, the prover's message is completely hidden.

The compiler works as follows. The parties emulate the original two-message protocol for explainable verifiers. The verifier also sends the first message of a conditional disclosure protocol for the statement $x^{\prime}$ asserting that its message is explainable (namely, it is in the support of the honest verifier's messages). The prover then responds with an encryption, relative to $x^{\prime}$, of its second message in the underlying protocol. The verifier decrypts and verifies the underlying protocol.

Intuitively, if the verifier does not behave in an explainable manner, the statement $x^{\prime}$ is false, the prover's message is hidden, and the verifier learns nothing. If the verifier is explainable, then the weak zero-knowledge guarantee of the underlying protocol kicks in. To argue soundness, we would like to give a reduction that can efficiently extract the prover's encrypted message, without using the verifier's randomness. To this end, the prover provides a proofof-knowledge of its message. To enable such a proof of knowledge in only two messages, we again rely on complexity leveraging.

A three-message compiler based on polynomial assumptions. A natural approach toward a three-message compiler based on polynomial assumptions is to augment the previous compiler with a three-message proof of knowledge (rather than a two-message one based on complexity leveraging). However, we do not know how to prove that this approach works. In a nutshell, the issue is that the explainability of the verifier's message may now depend on the first prover message, and cannot be efficiently tested. Instead, we take a different approach inspired by [18].

To understand the basic idea behind the compiler, imagine first that the language $\mathcal{L}$ is in NP $\cap$ coNP. The compiler works as follows. Given the statement $x$, the verifier provides, together with its message, a witness-indistinguishable argument that either $x \notin \mathcal{L}$ or that its message is explainable; namely, there exists randomness 
for the honest verifier strategy that is consistent with the messages. Note that $x \notin \mathcal{L}$ is indeed an NP statement since $\mathcal{L} \in$ coNP.

We first argue that the compiler preserves the privacy guarantee of the original protocol. By the soundness of the witnessindistinguishable argument, for every $x \in \mathcal{L}$, if the verifier sends a message that is not explainable, the prover immediately aborts. Thus, the view of a malicious verifier can be simulated from that of an explainable verifier. As for soundness, if $x \notin \mathcal{L}$, then, since $\mathcal{L} \in \mathbf{c o N P}$, there exists a witness for this fact. Given this witness as a non-uniform advice, the reduction can turn any cheating prover against the compiled scheme into a cheating prover against the original scheme. By witness indistinguishability, the reduction can use the witness for $x \notin \mathcal{L}$ to compute the witness-indistinguishable arguments without compromising the verifier's randomness.

To extend the above to all of NP, we use the first prover message to map the statement $x$ into a related coNP statement. For this, we rely on perfectly binding dense commitments where every string is a valid commitment to some value. The prover, in the first message, commits to the witness $w$ using the dense commitment. The verifier proceeds to prove that the prover's commitment can be opened to a string which is not a valid witness for $x$ (or that its messages can be explained).

To argue soundness when $x \notin \mathcal{L}$, note that the dense commitment can necessarily be opened to some string, which is not a witness. The reduction then proceeds as in the previous protocol Weak zero-knowledge follows by a simple extension of the previous argument. If the commitment in the first message indeed contains a valid witness, then by the binding of the commitment and the soundness of the verifier's proofs, the verifier fails to prove that the commitment is to a non-witness. Thus, the view of a malicious verifier can be simulated given the view of an explainable verifier and a commitment to a witness. Furthermore, by the hiding of the commitment, such simulation is possible even given a commitment to garbage, which the simulator can generate alone.

We note that this compiler actually preserves all natural security notions (like, zero-knowledge, weak zero-knowledge, or witness hiding).

Two-message witness-hiding compilers. We provide another twomessage compiler for witness hiding protocol that is based on polynomial assumptions and is also publicly verifiable. The compiler works for any language $\mathcal{L} \in \mathbf{N P}$ provided a witness encryption scheme for $\mathcal{L}$. In a witness encryption scheme for $\mathcal{L}$, it is possible to encrypt messages using statements $x$ as a public-key. Decryption can be done by anyone in possession of a corresponding witness $w$. In contrast, for $x \notin \mathcal{L}$, the encryption completely hides the message.

The compiler is as follows. Given the statement $x$, the verifier sends, together with its message, an encryption of its randomness under witness encryption, using the instance $x$ as the public-key. The honest prover, holding a witness, can decrypt and abort in case of malicious behavior. The compiler guarantees that if the verifier is not explainable, the prover aborts. Therefore, intuitively, such a verifier does not obtain any information. However, this intuition is misleading - a malicious verifier may generate messages without knowing whether they are explainable, and use the prover's abort decision to learn this bit of information. ${ }^{4}$ Nonetheless, the compiler does preserve witness hiding - the witness-finding reduction can simply guess if the verifier's message is explainable and simulate the prover's message accordingly. This only decreases its success probability by a factor of two.

\subsection{More on Related Work}

We next address related work in more detail.

Weak zero-knowledge and witness hiding. The notion of weak zero-knowledge is introduced in [32] who study the connection between 3-message public-coin weak zero-knowledge and so-called magic functions. (They also consider several variants of the definition.) The notion of witness hiding is introduced in [34] who prove that any witness-indistinguishable protocol is witness hiding for distributions on statements with at least two "independent" witnesses. In [16], three-message weak zero-knowledge and witness-hiding protocols are constructed based on non-standard assumptions, which by now are considered implausible [10, 24]. Specifically, these constructions are based on the notion of recognizable auxiliary-input point obfuscation that was shown in [10] to be impossible assuming virtual-grey-box obfuscation exists.

Upgrading weak zero-knowledge. The work of [27] considers two relaxed notions of zero-knowledge and proves that they are equivalent to their weak variants (where the simulator can depend on the distinguisher). The first notion they consider is distributional zero-knowledge where instances are sampled from some known distribution (and the simulator can depend on this distribution). The second notion is zero-knowledge against uniform distinguishers (these distinguishers can still get an auxiliary input, but the same input is given to the simulator). In both settings, the equivalence is shown for $(t, \epsilon)$-zero-knowledge, where the distinguisher's running time and distinguishing gap are bounded by $t$ and $\epsilon$, respectively, and the simulator's running time can depend on $t$ and $1 / \epsilon$.

Combining our weak-zero-knowledge protocols with the equivalence theorems of [27] yields distributional $(t, \epsilon)$-zero-knowledge and $(t, \epsilon)$-zero-knowledge against uniform distinguishers. Note that the two-message lower bound of [42] does not apply for these notions.

Distributional security against non-adaptive verifiers. The work of [53] constructs distributional weak-zero-knowledge and witnesshiding protocols for a restricted class of non-adaptive verifiers who choose their messages obliviously of the proven statement. They give protocols in three messages under standard assumptions, and in two messages under standard, but super-polynomial, assumptions. Their simulators and (witness-finding) reductions access the verifier as a black box.

Bounded description adversaries. Another type of relaxation considered in the literature is restricting the (adversarial) verifier or prover to a-priori bounded description (and arbitrary polynomial running time). Here (full-fledged) zero-knowledge can be constructed in two messages against bounded-description verifiers under standard, but super-polynomial, assumptions [13], and in

\footnotetext{
${ }^{4}$ In fact, for some instantiations of the witness encryption, this protocol reveals an arbitrary bit of the witness [18].
} 
three messages against bounded-description provers assuming also keyless hash functions that are collision-resistant against boundeddescription adversaries [11].

Super-polynomial simulation. Zero knowledge with simulators that run in super-polynomial time can be constructed in two messages from standard, but super-polynomial, assumptions $[4,57]$. One-message zero-knowledge with super-polynomial simulation can be constructed against uniform provers, assuming uniform collision-resistant keyless hash functions [7], or against non-uniform verifiers, but with weak soundness, assuming multi-collision-resistant keyless hash functions [15]. Such zeroknowledge implies a weak notion of witness hiding for distributions on instances where it is hard to find a witness, even for algorithms that run in the same super-polynomial time as the simulator.

Zero-knowledge proofs. So far, we have focused on the notion of arguments (which are only computationally sound). The round complexity of zero-knowledge proofs (which are statistically sound) has also been studied extensively. Four-message proofs are impossible to achieve via black-box simulation, except for languages in NP $\cap$ coMA [54]. Four message proofs with non-black-box simulation are only known assuming multi-collision-resistance keyless hash functions [14]. Recent evidence [35] suggests that, differently from zero-knowledge arguments, zero-knowledge proofs may be impossible to achieve in three messages (even with non-black-box simulation).

Honest-verifier zero-knowledge. For languages in the class SZK, there exist two-message proofs with an inefficient prover (known as Arthur-Merlin proofs) that are zero-knowledge only against honest verifiers $[1,58]$. Two-message honest-verifier zero-knowledge is implied by any NIZK with a common random string (such a NIZK exists under computational assumptions).

\section{DEFINITIONS}

In what follows, we denote by $\langle\mathrm{P}, \mathrm{V}\rangle$ a protocol between two parties $\mathrm{P}$ and $\mathrm{V}$. For input $w$ for $\mathrm{P}$, and common input $x$, we denote by $\mathrm{OUT}_{\mathrm{V}}\langle\mathrm{P}(w), \mathrm{V}\rangle(x)$ the output of $\mathrm{V}$ in the protocol. For honest verifiers, this output will be a single bit indicating acceptance (or rejection), malicious verifiers may have arbitrary output. Throughout, we assume that honest parties in all protocols are uniform PPT algorithms.

Definition 2.1 (Argument). A protocol $\langle\mathrm{P}, \mathrm{V}\rangle$ for an $\mathbf{N P}$ relation $\mathcal{R}_{\mathcal{L}}(x, w)$ is an argument if it satisfies:

(1) Completeness: For any $\lambda \in \mathbb{N}, x \in \mathcal{L} \cap\{0,1\}^{\lambda}, w \in \mathcal{R}_{\mathcal{L}}(x)$ :

$$
\operatorname{Pr}\left[\mathrm{OUT}_{\mathrm{V}}\langle\mathrm{P}(w), \mathrm{V}\rangle(x)=1\right]=1 .
$$

(2) Computational soundness: For any polynomial-size prover $\mathrm{P}^{*}=\left\{\mathrm{P}_{\lambda}^{*}\right\}_{\lambda}$, there exists a negligible $\mu$ such that for any security parameter $\lambda \in \mathbb{N}$, and any $x \in\{0,1\}^{\lambda} \backslash \mathcal{L}$,

$$
\operatorname{Pr}\left[\operatorname{OUT}_{\mathrm{V}}\left\langle\mathrm{P}_{\lambda}^{*}, \mathrm{~V}\right\rangle(x)=1\right] \leq \mu(\lambda) .
$$

The argument is sound against quasipoly provers, if the above holds also for quasipolynomial-size $\mathrm{P}^{*}$.
Remark 2.1 (Public verification). We say that the protocol is publicly verifiable if the verifier's decision bit can be computed from the protocol's messages (without verifier private state).

Remark 2.2 (Proofs). We say that the protocol is a proof if the soundness condition also holds against unbounded provers $\mathrm{P}^{*}$.

Remark 2.3 (Randomized provers). We assume that (adversarial) provers are deterministic. As usual, this is w.l.o.g (by fixing their coins to the ones that maximize their success probability).

\subsection{Weak Zero-Knowledge}

Definition 2.2 (WZK). A protocol $\langle\mathrm{P}, \mathrm{V}\rangle$ is WZK if there exists a PPT simulator $\mathrm{S}$, such that for any polynomial-size verifier $\mathrm{V}^{*}=$ $\left\{\mathrm{V}_{\lambda}^{*}\right\}_{\lambda}$, distinguisher $\mathrm{D}=\left\{\mathrm{D}_{\lambda}\right\}_{\lambda}$, and noticeable $\varepsilon(\lambda)$, there exists a negligible $\mu(\lambda)$, such that for any $\lambda \in \mathbb{N}, x \in \mathcal{L} \cap\{0,1\}^{\lambda}$, and $w \in \mathcal{R}_{\mathcal{L}}(x)$,

$$
\mathrm{OUT}_{\mathrm{V}_{\lambda}^{*}}\left\langle\mathrm{P}(w), \mathrm{V}_{\lambda}^{*}\right\rangle(x) \approx_{\mathrm{D}_{\lambda}, \varepsilon+\mu} \mathrm{S}\left(x, \mathrm{~V}_{\lambda}^{*}, \mathrm{D}_{\lambda}, 1^{1 / \varepsilon}\right)
$$

Remark 2.4 (w.l.o.g). When convenient we assume w.l.o.g that $\mathrm{V}^{*}$ is deterministic. This is as in the case of (standard) zero knowledge with a universal simulator [42]. We do not assume that the distinguisher is deterministic. This is in contrast to standard zeroknowledge where we often fix the best random coins for the distinguisher. In weak zero-knowledge this is not possible, as the simulated distribution can depend on the distinguisher.

Also, when convenient we assume w.l.o.g that verifiers always output their entire view consisting of the prover message and, if they are probabilistic, their randomness.

\subsection{Witness Hiding}

Definition 2.3 (WH). A protocol $\langle\mathrm{P}, \mathrm{V}\rangle$ is WH if there exists a PPT reduction $\mathrm{R}$, such that for any polynomial-size verifier $\mathrm{V}^{*}=\left\{\mathrm{V}_{\lambda}^{*}\right\}_{\lambda}$ and noticeable $\varepsilon(\lambda)$, there exists a negligible $\mu(\lambda)$, such that for any $\lambda \in \mathbb{N}$ and $x \in \mathcal{L} \cap\{0,1\}^{\lambda}$,

$$
\begin{gathered}
\operatorname{Pr}\left[\mathrm{OUT}_{\mathrm{V}_{\lambda}^{*}}\left\langle\mathrm{P}(w), \mathrm{V}_{\lambda}^{*}\right\rangle(x) \in \mathcal{R}_{\mathcal{L}}(x)\right] \leq \\
\operatorname{Pr}\left[\mathrm{R}\left(x, \mathrm{~V}_{\lambda}^{*}, 1^{1 / \varepsilon}\right) \in \mathcal{R}_{\mathcal{L}}(x)\right]+\varepsilon(\lambda)+\mu(\lambda) .
\end{gathered}
$$

Remark 2.5 (Randomized verifiers). As in Remark 2.4, and for the same reasons, the above definition considers w.l.o.g only deterministic verifiers $\mathrm{V}^{*}$.

It is well-known that WZK implies WH (by considering the specific distinguishers that checks if the verifier's output is a valid witness).

\subsection{Explainable Verifiers}

Roughly speaking, explainable verifiers are verifiers whose messages are (almost) always in the support of the honest verifier's messages, regardless of how the prover's messages are generated. We also allow such verifiers to abort at any stage.

DeFinition 2.4 (EXPLAINABle TRANSCRIPT). Let $\langle\mathrm{P}, \mathrm{V}\rangle$ be a protocol, $\mathrm{P}^{*}$ be an arbitrary prover, and $\mathrm{V}^{*}$ an arbitrary verifier. We say that a transcript $T$ of an execution $\left\langle\mathrm{P}^{*}, \mathrm{~V}^{*}\right\rangle(x)$ is explainable if there exists honest verifier coins $r$ such that $T$ is consistent with the 
transcript of an execution $\left\langle\mathrm{P}^{*}, \mathrm{~V}_{r}\right\rangle$ until the point in $T$ that $\mathrm{V}^{*}$ aborts. (Here $\mathrm{V}_{r}$ is the honest verifier using coins $r$ ).

DEFINITION 2.5 (EXPLAINABLE VERIFIER). Let $\langle\mathrm{P}, \mathrm{V}\rangle$ be a protocol. $A$ (possibly probabilistic) verifier $\mathrm{V}^{*}=\left\{\mathrm{V}_{\lambda}^{*}\right\}$ is explainable if there exists a negligible $\mu(\lambda)$ such that for any prover $\mathrm{P}^{*}, \lambda \in \mathbb{N}$, and $x \in\{0,1\}^{\lambda}$,

$$
\underset{\mathrm{V}_{\lambda}^{*}}{\operatorname{Pr}}\left[T \text { is explainable } \mid T \leftarrow\left\langle\mathrm{P}^{*}, \mathrm{~V}_{\lambda}^{*}\right\rangle(x)\right] \geq 1-\mu(\lambda) .
$$

In the two-message setting, We will also consider a simpler notion of always explainable verifiers, which are deterministic verifiers that always output a message in the support of the honest verifier.

Definition 2.6 (Always-eXPLAINABle Verifier). A deterministic verifier $\mathrm{V}^{*}=\left\{\mathrm{V}_{\lambda}^{*}\right\}_{\lambda}$ is always explainable if for any prover $\mathrm{P}^{*}$, $\lambda \in \mathbb{N}, x \in\{0,1\}^{\lambda}$, and $T=\left\langle\mathrm{P}^{*}, \mathrm{~V}_{\lambda}^{*}\right\rangle(x), T$ is explainable and is not $\perp$.

Explainable WZK and WH. WZK and WH against explainable verifiers are defined exactly as WZK and WH only that the (respective) definition only holds against explainable verifiers rather than all verifiers. We also note that WZK $\Longrightarrow \mathrm{WH}$ also holds for explainable verifiers.

Remark 2.6 (w.l.o.g). We note that in the two-message setting, we can assume w.l.o.g that explainable verifiers are always explainable. This is because aborts can be easily simulated and malicious verifier messages (that are not abort) occur with negligible probability.

\subsection{Witness Indistinguishability}

We consider two-message witness-indistinguishable (WI) arguments with delayed input, where the prover obtains the input statement and witness after the first round of the protocol. In some of our protocols, we will also require a weak argument of knowledge property that says that there exists a quasipolynomial-time witness extractor

Definition 2.7 (Two-MESSAGE ARgument with DELAYED INPUT). A two-message protocol $\langle\mathrm{P}, \mathrm{V}\rangle$ is a delayed input WI argument if $\mathrm{V}$ consists of two PPT algorithms $\left(\mathrm{V}_{1}, \mathrm{~V}_{2}\right)$ that satisfy:

(1) Completeness: For any $\lambda \in \mathbb{N}, x \in \mathcal{L} \cap\{0,1\}^{\lambda}, w \in \mathcal{R}_{\mathcal{L}}(x)$ :

$$
\operatorname{Pr}\left[\begin{array}{l|l}
\mathrm{V}_{2}\left(x, \mathrm{wi}_{2} ; \tau\right)=1 & \begin{array}{l}
\left(\mathrm{wi}_{1}, \tau\right) \leftarrow \mathrm{V}_{1}\left(1^{\lambda}\right) \\
\mathrm{wi}_{2} \leftarrow \mathrm{P}\left(x, w, \mathrm{wi}_{1}\right)
\end{array}
\end{array}\right]=1 .
$$

(2) Computational soundness: For any polynomial-size prover $\mathrm{P}^{*}=\left\{\mathrm{P}_{\lambda}^{*}\right\}_{\lambda}$, there exists a negligible $\mu$ such that for any security parameter $\lambda \in \mathbb{N}$,

$$
\operatorname{Pr}\left[\begin{array}{c|c}
\mathrm{V}_{2}\left(x, \mathrm{wi}_{2} ; \tau\right)=1 & \left(\mathrm{wi}_{1}, \tau\right) \leftarrow \mathrm{V}_{1}\left(1^{\lambda}\right) \\
x \in\{0,1\}^{\lambda} \backslash \mathcal{L} & \left(x, \mathrm{wi}_{2}\right) \leftarrow \mathrm{P}^{*}\left(\mathrm{wi}_{1}\right)
\end{array}\right] \leq \mu(\lambda) .
$$

(3) Witness indistinguishability: For any polynomial-size verifier $\mathrm{V}^{*}=\left\{\mathrm{V}_{\lambda}^{*}\right\}_{\lambda}$, there exists a negligible $\mu$ such that for any security parameter $\lambda \in \mathbb{N}$,

$$
\operatorname{Pr}\left[\begin{array}{l|l}
\mathrm{V}_{\lambda}^{*}\left(\mathrm{wi}_{2}\right)=b & \left(\mathrm{wi}_{1}, x, w_{0}, w_{1}\right) \leftarrow \mathrm{V}_{\lambda}^{*} \\
x \in \mathcal{L} \cap\{0,1\}^{\lambda} & b \leftarrow\{0,1\} \\
w_{0}, w_{1} \in \mathcal{R}_{\mathcal{L}}(x) & \mathrm{wi}_{2} \leftarrow \mathrm{P}\left(x, w_{b}, \mathrm{wi}_{1}\right)
\end{array}\right] \leq \frac{1}{2}+\mu(\lambda) .
$$

The argument has a quasipolynomial witness extractor if there exists a quasipolynomial time extractor $\mathrm{E}$ such that for any polynomial-size prover $\mathrm{P}^{*}=\left\{\mathrm{P}_{\lambda}^{*}\right\}_{\lambda}$, there exists a negligible $\mu$ such that for any security parameter $\lambda \in \mathbb{N}$,

$$
\operatorname{Pr}\left[\begin{array}{l|l}
\mathrm{V}_{2}\left(x, \mathrm{wi}_{2} ; \tau\right)=1 & \left(\mathrm{wi}_{1}, \tau\right) \leftarrow \mathrm{V}_{1}\left(1^{\lambda}\right) \\
w \notin \mathcal{R}_{\mathcal{L}}(x) & \left(x, \mathrm{wi}_{2}\right) \leftarrow \mathrm{P}^{*}\left(\mathrm{wi}_{1}\right) \\
& w \leftarrow \mathrm{E}\left(x, \mathrm{wi}_{1}, \mathrm{wi}_{2}\right)
\end{array}\right] \leq \mu(\lambda)
$$

Instantiations. Two-message WI arguments with delayed input that are also publicly verifiable (and are in fact also proofs) can be based either on trapdoor permutations [31], standard assumptions on bilinear groups [47], or indistinguishability obfuscation [18]. Such privately-verifiable arguments can be constructed from any 2-message oblivious transfer against malicious receivers and superpolynomial semi-honest senders $[4,53]$. In particular, they can be constructed from (super-polynomial) LWE [22].

A two-message WI argument with delayed input and a quasipolynomial witness extractor can be constructed from any (plain) twomessage WI argument and subexponentially-secure two-message commitments [57], which in turn can be constructed from subexponentially secure one-way functions $[51,55]$.

\section{WEAK ZK AGAINST EXPLAINABLE VERIFIERS}

In this section, we construct WZK protocols against explainable verifiers. We start with the three-message protocol and then move to the 2-message protocol, which will be a special case of the 3message protocol.

\subsection{The Three-Message Protocol}

In this section, we construct a three-message WZK argument against explainable verifiers.

Ingredients and notation: We rely on the following primitives, complete definitions of which can be found in the full version of the paper.

- A 3-message extractable commitment (EC.S, EC.R, EC.V). We denote its messages by $\left(c_{1}, c_{2}, c_{3}\right)$.

- a 2-message WI argument (WI.P, WI.V) with delayed input. We denote its messages by $\left(\mathrm{wi}_{1}, \mathrm{wi}_{2}\right)$.

- A non-interactive perfectly-binding commitment Com.

- A fully-homomorphic encryption scheme (FHE.Enc, FHE.Dec, FHE.Eval).

- A compute-and-compare obfuscator $O$.

- A random self-reducible public-key encryption (RSR.Gen, RSR.Enc, RSR.Dec, RSR. $\widetilde{\text { Dec }}$ ). (In fact, relaxed RSR suffices, refer to the full version.)

We describe the protocol in Figure 1 and defer security analysis to the full version of the paper. 


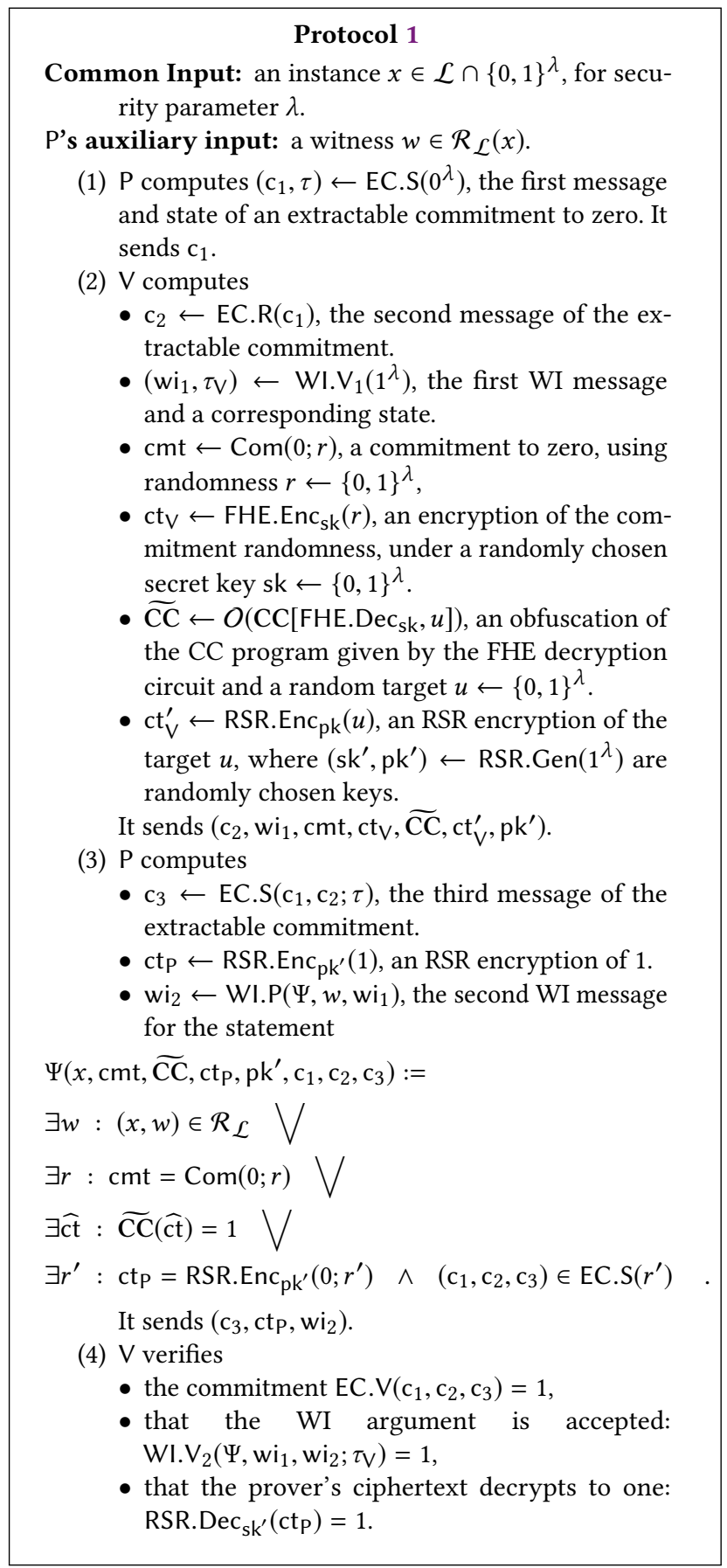

Figure 1: A 3-message WZK argument for NP against explainable verifiers.

\subsection{The Two-Message Protocol}

We also construct, under stronger assumptions, a two-message WZK argument against explainable verifiers and quasipolynomial provers. Specifically we strengthen the ingredients as follows.

\section{Ingredients and notation:}

- A 2-message extractable commitment (EC.S, EC.R, EC.V) with a quasipolynomial extractor. We denote its messages by $\left(c_{2}, c_{3}\right)$; this is for consistency with the three-message protocol ( $\mathrm{c}_{1}$ is always empty).

- a 2-message WI argument (WI.P, WI.V) with delayed input, sound against quasipolynomial provers.

- A non-interactive perfectly-binding commitment scheme Com, hiding against quasipolynomial distinguishers.

- A fully-homomorphic encryption scheme (FHE.Enc, FHE.Dec, FHE.Eval), secure against quasipolynomial distinguishers.

- A compute-and-compare obfuscator $O$, secure against quasipolynomial distinguishers.

- A random self-reducible public-key encryption (RSR.Gen, RSR.Enc, RSR.Dec, RSR. $\widetilde{D e c}$ ), secure against quasipolynomial distinguishers. (In fact, relaxed RSR suffices. To simplify the description of the protocol, we rely on standard RSR, and later remark why relaxed RSR suffices.)

The protocol is identical to the one in Figure 1, only that since the first commitment message $c_{1}$ is always empty, it consists of only two messages. The proof is essentially identical to that of the previous protocol, only taking into accountthe above enhancements. In the proof of soundness, the prover $\mathrm{P}^{*}$ and the extractor $\mathrm{E}$ are now quasipolynomial instead of polynomial. Thus, each of the reductions in the proof is now quasipolynomial instead of polynomial, and breaks the quasipolynomial (rather than polynomial) security of the underlying primitives. The same WZK proof applies (and in fact becomes even simpler in the two-message setting).

\section{FROM EXPLAINABLE TO MALICIOUS VERIFIERS}

In this section, we present generic transformations that compile protocols that are private (according to some natural notion, such as $\mathrm{ZK}, \mathrm{WZK}, \mathrm{WH}$ ) against explainable verifiers into ones that satisfy the same privacy guarantee against malicious verifiers. We defer analyses to the full version.

These transformations include the following:

- A 3-message transformation that preserves WZK (or ZK), based on polynomial hardness assumptions.

- A 2-message transformation that preserves WZK, based on super-polynomial hardness assumptions.

- A 2-message transformation that preserves WH, based on polynomial witness encryption.

\subsection{The Three-Message Transformation}

We provide a transformation that compiles any 3-message WZK protocol against explainable verifiers into one against malicious verifiers.

Ingredients and notation:

- A 2-message WI argument for NP with delayed input (WI.P, WI.V). We denote its messages by $\left(\mathrm{wi}_{1}, \mathrm{wi}_{2}\right)$.

- A non-interactive dense commitment scheme Com. 
- A 3-message argument system $\langle\mathrm{P}, \mathrm{V}\rangle$ for an NP language $\mathcal{L}$ that is WZK against explainable verifiers. We denote its messages by $\left(\arg _{1}, \arg _{2}, \arg _{3}\right)$.

We describe the protocol in Figure 2.

\footnotetext{
Protocol 2

Common Input: an instance $x \in \mathcal{L} \cap\{0,1\}^{\lambda}$, for security parameter $\lambda$.

P's auxiliary input: a witness $w \in \mathcal{R}_{\mathcal{L}}(x)$.

(1) $\bar{P}$ computes

- $\arg _{1}$, the first message in the original protocol.

- $\mathrm{cmt} \leftarrow \operatorname{Com}(w)$ a commitment to the witness.

- $\mathrm{wi}_{1}$, the first message of a WI argument.

It sends $\left(\arg _{1}, \mathrm{cmt}, \mathrm{wi}_{1}\right)$.

(2) $\bar{V}$ computes

- $\arg _{2}$, the second message in the original protocol.

- $\mathrm{wi}_{2}$, the second WI message for the statement $\Phi\left(x, \mathrm{cmt}, \arg _{1}, \arg _{2}\right): \exists r: \arg _{2}=\mathrm{V}\left(x, \arg _{1} ; r\right) \vee$ $\exists r, s: \mathrm{cmt}=\operatorname{Com}(s ; r), s \notin \mathcal{R}_{\mathcal{L}}(x)$.

It sends $\left(\arg _{2}, \mathrm{wi}_{2}\right)$.

(3) $\bar{P}$ verifies the WI argument $\left(w_{1} i_{1}, w_{2}\right)$ for the statement $\Phi$, and aborts if it does not accept.

It then computes $\arg _{3}$, the third message in the original protocol, and sends it.

(4) $\bar{V}$ verifies the argument $\left(\arg _{1}, \arg _{2}, \arg _{3}\right)$.
}

Figure 2: A WZK argument $\langle\bar{P}, \bar{V}\rangle$ for NP against malicious verifiers.

\subsection{The Two-Message Transformation}

We provide a transformation that compiles any 2-message WZK protocol against explainable verifiers with soundness against quasipolynomial provers (like the one from Section 3.2) into one against malicious verifiers.

\section{Ingredients and notation:}

- A 2-message WI argument for NP (delayed input) with a quasipolynomial witness extractor $\left\langle\right.$ WI.P, $\left.\left(\mathrm{WI} . \mathrm{V}_{1}, \mathrm{WI}_{\mathrm{V}} \mathrm{V}_{2}\right)\right\rangle$. We denote its messages by $\left(\mathrm{wi}_{1}, \mathrm{wi}_{2}\right)$.

- Conditional disclosure of secrets (CDS.R, CDS.S, CDS.D) for NP, with receiver simulation security against quasipolynomial time senders.

- A 2-message argument system $\langle\mathrm{P}, \mathrm{V}\rangle$ for an NP language $\mathcal{L}$ that is WZK against explainable verifiers and sound against quasipolynomial provers. We denote its messages by $\left(\arg _{1}, \arg _{2}\right)$.

We describe the protocol in Figure 3.

\subsection{The Two-Message Public-Coin WH Transformation}

This protocol can be viewed as a variant of Protocol 2, where the verifier, rather than using a WI system to prove that it behaves

\section{Protocol 3}

Common Input: an instance $x \in \mathcal{L} \cap\{0,1\}^{\lambda}$, for security parameter $\lambda$.

P's auxiliary input: a witness $w \in \mathcal{R}_{\mathcal{L}}(x)$.

(1) $\bar{V}$ computes

- $\left(\mathrm{wi}_{1}, \tau \mathrm{V}\right) \leftarrow \mathrm{WI} . \mathrm{V}_{1}\left(1^{\lambda}\right)$, the first WI message.

- $\arg _{1}$, the verifier message in the original protocol $\langle\mathrm{P}, \mathrm{V}\rangle(x)$. It stores the randomness $r$ used by the verier to generate the message.

- $\left(\mathrm{ct}_{\mathrm{R}}, \mathrm{k}\right) \leftarrow \mathrm{CDS} \cdot \mathrm{R}(\Psi, r)$, a CDS receiver message for the statement $\Psi\left(\arg _{1}\right)$ attesting that $\arg _{1}$ was computed according to the honest verifier $\mathrm{V}$, using $r$ as the witness.

It sends $\left(\mathrm{wi}_{1}, \arg _{1}, \mathrm{ct}_{\mathrm{R}}\right)$.

(2) $\bar{P}$ computes

- $\arg _{2}$, the prover message in the original protocol $\langle\mathrm{P}, \mathrm{V}\rangle$.

- ct $_{\mathrm{S}} \leftarrow \mathrm{CDS} . \mathrm{S}\left(\Psi, \arg _{2}, \mathrm{ct}_{\mathrm{R}}\right)$, a CDS sender message encrypting $\arg _{2}$.

- $\mathrm{wi}_{2} \leftarrow$ WI.P $\left(\Phi, \arg _{2}, \mathrm{wi}_{1}\right)$, the second WI message for the statement

$\Phi\left(x, \Psi, \mathrm{ct}_{\mathrm{S}}, \mathrm{ct}_{\mathrm{R}}\right):=\exists \arg : \mathrm{ct}_{\mathrm{S}} \in \mathrm{CDS} . \mathrm{S}\left(\Psi, \arg , \mathrm{ct}_{\mathrm{R}}\right) \vee x \in \mathcal{L}$.

It sends (cts, wi 2 ).

(3) $\bar{V}$ then

- Runs WI. $\mathrm{V}_{2}\left(\Phi, \mathrm{wi}_{1}, \mathrm{wi}_{2} ; \tau_{\mathrm{V}}\right)$ to verify the WI argument for the statement $\Phi$.

- Decrypts $\widetilde{\arg }_{2} \leftarrow$ CDS.D (cts $\left._{\mathrm{S}}\right)$.

- Verifies the original argument $\left(\arg _{1}, \widetilde{\arg }_{2}\right)$.

Figure 3: A WZK argument $\langle\bar{P}, \bar{V}\rangle$ for NP against malicious verifiers.

honestly, proves it using a witness encryption of its coins under $x$. This proof is simulatable if $x \notin \mathcal{L}$, and otherwise is sound. We defer a complete description to the full version of the paper.

\section{ACKNOWLEDGMENTS}

N. Bitansky is supported by the Alon Young Faculty Fellowship, by Len Blavatnik and the Blavatnik Family foundation, and an ISF grant 18/484. O. Paneth is supported by NSF Grants CNS-1350619 and CNS-1414119, and the Defense Advanced Research Projects Agency (DARPA) and the U.S. Army Research Office under contracts W911NF-15-C-0226 and W911NF-15-C-0236.Any opinions, findings and conclusions or recommendations expressed are those of the author(s) and do not necessarily reflect the views of the DARPA and ARO.

\section{REFERENCES}

[1] William Aiello and Johan Håstad. 1991. Statistical Zero-Knowledge Languages can be Recognized in Two Rounds. 7. Comput. Syst. Sci. 42, 3 (1991), 327-345. https://doi.org/10.1016/0022-0000(91)90006-Q

[2] William Aiello, Yuval Ishai, and Omer Reingold. 2001. Priced Oblivious Transfer: How to Sell Digital Goods. In EUROCRYPT (Lecture Notes in Computer Science), Vol. 2045. Springer, 119-135.

[3] Prabhanjan Ananth and Abhishek Jain. 2017. On Secure Two-Party Computation in Three Rounds. In Theory of Cryptography - 15th International Conference, TCC 
2017, Baltimore, MD, USA, November 12-15, 2017, Proceedings, Part I. 612-644 https://doi.org/10.1007/978-3-319-70500-2_21

[4] Saikrishna Badrinarayanan, Sanjam Garg, Yuval Ishai, Amit Sahai, and Akshay Wadia. 2017. Two-Message Witness Indistinguishability and Secure Computation in the Plain Model from New Assumptions. In Advances in Cryptology ASIACRYPT 2017. 275-303. https://doi.org/10.1007/978-3-319-70700-6_10

[5] Boaz Barak. 2001. How to Go Beyond the Black-Box Simulation Barrier. In 42nd Annual Symposium on Foundations of Computer Science, FOCS 2001, 14-17 October 2001, Las Vegas, Nevada, USA. 106-115. https://doi.org/10.1109/SFCS.2001.959885

[6] Boaz Barak, Shien Jin Ong, and Salil P. Vadhan. 2007. Derandomization in Cryptography. SIAM f. Comput. 37, 2 (2007), 380-400. https://doi.org/10.1137/ 050641958

[7] Boaz Barak and Rafael Pass. 2004. On the Possibility of One-Message Weak ZeroKnowledge. In Theory of Cryptography, First Theory of Cryptography Conference, TCC 2004, Cambridge, MA, USA, February 19-21, 2004, Proceedings. 121-132. https: //doi.org/10.1007/978-3-540-24638-1_7

[8] Mihir Bellare, Markus Jakobsson, and Moti Yung. 1997. Round-Optimal ZeroKnowledge Arguments Based on any One-Way Function. In Advances in Cryptology - EUROCRYPT '97. 280-305. https://doi.org/10.1007/3-540-69053- 020

[9] Mihir Bellare and Adriana Palacio. 2004. Towards Plaintext-Aware Public-Key Encryption Without Random Oracles. In ASIACRYPT. 48-62.

[10] Mihir Bellare, Igors Stepanovs, and Stefano Tessaro. 2016. Contention in Cryptoland: Obfuscation, Leakage and UCE. In Theory of Cryptography - 13th International Conference, TCC 2016-A, Tel Aviv, Israel, fanuary 10-13, 2016, Proceedings, Part II. 542-564. https://doi.org/10.1007/978-3-662-49099-0_20

[11] Nir Bitansky, Zvika Brakerski, Yael Tauman Kalai, Omer Paneth, and Vinod Vaikuntanathan. 2016. 3-Message Zero Knowledge Against Human Ignorance. In Theory of Cryptography - 14th International Conference, TCC 2016-B, Beijing, China, October 31 - November 3, 2016, Proceedings, Part I. 57-83. https://doi.org/ 10.1007/978-3-662-53641-4 3

[12] Nir Bitansky, Ran Canetti, Alessandro Chiesa, Shafi Goldwasser, Huijia Lin, Aviad Rubinstein, and Eran Tromer. 2017. The Hunting of the SNARK. F. Cryptology 30, 4 (2017), 989-1066. https://doi.org/10.1007/s00145-016-9241-9

[13] Nir Bitansky, Ran Canetti, Omer Paneth, and Alon Rosen. 2014. On the existence of extractable one-way functions. In Symposium on Theory of Computing, STOC 2014, New York, NY, USA, May 31 - June 03, 2014. 505-514. https://doi.org/10. $1145 / 2591796.2591859$

[14] Nir Bitansky, Yael Tauman Kalai, and Omer Paneth. 2018. Multi-collision resistance: a paradigm for keyless hash functions. In Proceedings of the 50th Annual ACM SIGACT Symposium on Theory of Computing, STOC 2018, Los Angeles, CA, USA, June 25-29, 2018. 671-684. https://doi.org/10.1145/3188745.3188870

[15] Nir Bitansky and Huijia Lin. 2018. One-Message Zero Knowledge and NonMalleable Commitments. In Theory of Cryptography Conference, TCC 2018, Goa, India, November 11-14, 2018, Proceedings.

[16] Nir Bitansky and Omer Paneth. 2012. Point Obfuscation and 3-Round ZeroKnowledge. In Theory of Cryptography - 9th Theory of Cryptography Conference, TCC 2012, Taormina, Sicily, Italy, March 19-21, 2012. Proceedings. 190-208. https: //doi.org/10.1007/978-3-642-28914-9_11

[17] Nir Bitansky and Omer Paneth. 2015. On Non-Black-Box Simulation and the Impossibility of Approximate Obfuscation. SIAM 7. Comput. 44, 5 (2015), 13251383. https://doi.org/10.1137/130928236

[18] Nir Bitansky and Omer Paneth. 2015. ZAPs and Non-Interactive Witness Indistinguishability from Indistinguishability Obfuscation. In Theory of Cryptography - 12th Theory of Cryptography Conference, TCC 2015, Warsaw, Poland, March 23-25, 2015, Proceedings, Part II. 401-427. https://doi.org/10.1007/978-3-662-46497-7_16

[19] Manuel Blum and Silvio Micali. 1984. How to Generate Cryptographically Strong Sequences of Pseudo-Random Bits. SIAM f. Comput. 13, 4 (1984), 850-864. https: //doi.org/10.1137/0213053

[20] Elette Boyle, Sanjam Garg, Abhishek Jain, Yael Tauman Kalai, and Amit Sahai 2013. Secure Computation against Adaptive Auxiliary Information. In Advances in Cryptology - CRYPTO 2013. 316-334. https://doi.org/10.1007/978-3-642-40041-4 18

[21] Elette Boyle and Rafael Pass. 2015. Limits of Extractability Assumptions with Distributional Auxiliary Input. In Advances in Cryptology - ASIACRYPT 2015 236-261. https://doi.org/10.1007/978-3-662-48800-3_10

[22] Zvika Brakerski and Nico Döttling. 2018. Two-Message Statistical Sender-Private OT from LWE. IACR Cryptology ePrint Archive 2018 (2018), 530. https://eprint. iacr.org/2018/530

[23] Zvika Brakerski and Vinod Vaikuntanathan. 2014. Efficient Fully Homomorphic Encryption from (Standard) \$\mathsf\{LWE\}\$. SIAM f. Comput. 43, 2 (2014), 831-871. https://doi.org/10.1137/120868669

[24] Christina Brzuska and Arno Mittelbach. 2014. Indistinguishability Obfuscation versus Multi-bit Point Obfuscation with Auxiliary Input. In Advances in Cryptology - ASIACRYPT 2014. 142-161. https://doi.org/10.1007/978-3-662-45608-8_8

[25] Yilei Chen, Vinod Vaikuntanathan, and Hoeteck Wee. 2018. GGH15 Beyond Permutation Branching Programs: Proofs, Attacks, and Candidates. In Advances in Cryptology - CRYPTO 2018. 577-607. https://doi.org/10.1007/978-3-319-96881-0_ 20
[26] Kai-Min Chung, Huijia Lin, and Rafael Pass. 2013. Constant-Round Concurrent Zero Knowledge from P-Certificates. In 54th Annual IEEE Symposium on Foundations of Computer Science, FOCS 2013, 26-29 October, 2013, Berkeley, CA, USA. 50-59. https://doi.org/10.1109/FOCS.2013.14

[27] Kai-Min Chung, Edward Lui, and Rafael Pass. 2015. From Weak to Strong ZeroKnowledge and Applications. In Theory of Cryptography - 12th Theory of Cryptography Conference, TCC 2015, Warsaw, Poland, March 23-25, 2015, Proceedings, Part I. 66-92. https://doi.org/10.1007/978-3-662-46494-6 4

[28] Kai-Min Chung, Rafael Pass, and Karn Seth. 2016. Non-Black-Box Simulation from One-Way Functions and Applications to Resettable Security. SIAM f. Comput. 45, 2 (2016), 415-458. https://doi.org/10.1137/130946083

[29] Ronald Cramer and Victor Shoup. 2002. Universal Hash Proofs and a Paradigm for Adaptive Chosen Ciphertext Secure Public-Key Encryption. In Advances in Cryptology - EUROCRYPT 2002. 45-64. https://doi.org/10.1007/3-540-46035-7_4

[30] Yi Deng, Vipul Goyal, and Amit Sahai. 2009. Resolving the Simultaneous Resettability Conjecture and a New Non-Black-Box Simulation Strategy. In 50th Annual IEEE Symposium on Foundations of Computer Science, FOCS 2009, October 25-27, 2009, Atlanta, Georgia, USA. 251-260. https://doi.org/10.1109/FOCS.2009.59

[31] Cynthia Dwork and Moni Naor. 2007. Zaps and Their Applications. SIAM 7. Comput. 36, 6 (2007), 1513-1543. https://doi.org/10.1137/S0097539703426817

[32] Cynthia Dwork, Moni Naor, Omer Reingold, and Larry J. Stockmeyer. 2003. Magic Functions. 7. ACM 50, 6 (2003), 852-921. https://doi.org/10.1145/950620.950623

[33] Uriel Feige, Dror Lapidot, and Adi Shamir. 1999. Multiple NonInteractive Zero Knowledge Proofs Under General Assumptions. SIAM J. Comput. 29, 1 (1999), 1-28. https://doi.org/10.1137/S0097539792230010

[34] Uriel Feige and Adi Shamir. 1990. Witness Indistinguishable and Witness Hiding Protocols. In Proceedings of the 22nd Annual ACM Symposium on Theory of Computing, May 13-17, 1990, Baltimore, Maryland, USA. 416-426. https: //doi.org/10.1145/100216.100272

[35] Nils Fleischhacker, Vipul Goyal, and Abhishek Jain. 2018. On the Existence of Three Round Zero-Knowledge Proofs. In Advances in Cryptology - EUROCRYPT 2018. 3-33. https://doi.org/10.1007/978-3-319-78372-7_1

[36] Sanjam Garg, Craig Gentry, Shai Halevi, Mariana Raykova, Amit Sahai, and Brent Waters. 2016. Candidate Indistinguishability Obfuscation and Functional Encryption for All Circuits. SIAM F. Comput. 45, 3 (2016), 882-929. https: //doi.org/10.1137/14095772X

[37] Sanjam Garg, Craig Gentry, Amit Sahai, and Brent Waters. 2013. Witness encryption and its applications. In Symposium on Theory of Computing Conference, STOC'13, Palo Alto, CA, USA, June 1-4, 2013. 467-476. https://doi.org/10.1145/ 2488608.2488667

[38] Craig Gentry. 2009. A fully homomorphic encryption scheme. Ph.D. Dissertation. Stanford University. crypto.stanford.edu/craig.

[39] Craig Gentry. 2009. Fully homomorphic encryption using ideal lattices. In Proceedings of the 41st Annual ACM Symposium on Theory of Computing, STOC 2009, Bethesda, MD, USA, May 31 - June 2, 2009. 169-178. https://doi.org/10.1145/ 1536414.1536440

[40] Oded Goldreich and Hugo Krawczyk. 1996. On the Composition of ZeroKnowledge Proof Systems. SIAM F. Comput. 25, 1 (1996), 169-192. https: //doi.org/10.1137/S0097539791220688

[41] Oded Goldreich, Silvio Micali, and Avi Wigderson. 1991. Proofs that Yield Nothing But Their Validity for All Languages in NP Have Zero-Knowledge Proof Systems. f. ACM 38, 3 (1991), 691-729. https://doi.org/10.1145/116825.116852

[42] Oded Goldreich and Yair Oren. 1994. Definitions and Properties of ZeroKnowledge Proof Systems. . Cryptology 7, 1 (1994), 1-32. https://doi.org/ 10.1007/BF00195207

[43] Shafi Goldwasser, Silvio Micali, and Charles Rackoff. 1989. The Knowledge Complexity of Interactive Proof Systems. SIAM 7. Comput. 18, 1 (1989), 186-208. https://doi.org/10.1137/0218012

[44] Rishab Goyal, Susan Hohenberger, Venkata Koppula, and Brent Waters. 2017. A Generic Approach to Constructing and Proving Verifiable Random Functions. In Theory of Cryptography - 15th International Conference, TCC 2017, Baltimore, MD, USA, November 12-15, 2017, Proceedings, Part II. 537-566. https://doi.org/10.1007/ 978-3-319-70503-3_18

[45] Rishab Goyal, Venkata Koppula, and Brent Waters. 2017. Lockable Obfuscation. In 58th IEEE Annual Symposium on Foundations of Computer Science, FOCS 2017, Berkeley, CA, USA, October 15-17, 2017. 612-621. https://doi.org/10.1109/FOCS. 2017.62

[46] Vipul Goyal. 2013. Non-black-box simulation in the fully concurrent setting. In Symposium on Theory of Computing Conference, STOC'13, Palo Alto, CA, USA, June 1-4, 2013. 221-230. https://doi.org/10.1145/2488608.2488637

[47] Jens Groth, Rafail Ostrovsky, and Amit Sahai. 2012. New Techniques for Noninteractive Zero-Knowledge. F. ACM 59, 3 (2012), 11:1-11:35. https: //doi.org/10.1145/2220357.2220358

[48] Satoshi Hada and Toshiaki Tanaka. 1998. On the Existence of 3-Round ZeroKnowledge Protocols. In Proceedings of the 18th Annual International Cryptology Conference. 408-423.

[49] Iftach Haitner, Yuval Ishai, Eyal Kushilevitz, Yehuda Lindell, and Erez Petrank. 2011. Black-Box Constructions of Protocols for Secure Computation. SIAM 7. 
Comput. 40, 2 (2011), 225-266. https://doi.org/10.1137/100790537

[50] Iftach Haitner, Alon Rosen, and Ronen Shaltiel. 2009. On the (Im)Possibility of Arthur-Merlin Witness Hiding Protocols. In Theory of Cryptography, 6th Theory of Cryptography Conference, TCC 2009, San Francisco, CA, USA, March 15-17, 2009. Proceedings. 220-237. https://doi.org/10.1007/978-3-642-00457-5_14

[51] Johan Håstad, Russell Impagliazzo, Leonid A. Levin, and Michael Luby. 1999. A Pseudorandom Generator from any One-way Function. SIAM f. Comput. 28, 4 (1999), 1364-1396.

[52] Pavel Hubáček and Daniel Wichs. 2015. On the Communication Complexity of Secure Function Evaluation with Long Output. In Proceedings of the 2015 Conference on Innovations in Theoretical Computer Science, ITCS 2015, Rehovot, Israel, fanuary 11-13, 2015. 163-172. https://doi.org/10.1145/2688073.2688105

[53] Abhishek Jain, Yael Tauman Kalai, Dakshita Khurana, and Ron Rothblum. 2017. Distinguisher-Dependent Simulation in Two Rounds and its Applications. In Advances in Cryptology - CRYPTO 2017. 158-189. https://doi.org/10.1007/ 978-3-319-63715-0_6

[54] Jonathan Katz. 2012. Which Languages Have 4-Round Zero-Knowledge Proofs? f. Cryptology 25, 1 (2012), 41-56. https://doi.org/10.1007/s00145-010-9081-y
[55] Moni Naor. 1991. Bit Commitment Using Pseudorandomness. 7. Cryptology 4, 2 (1991), 151-158.

[56] Rafail Ostrovsky, Anat Paskin-Cherniavsky, and Beni Paskin-Cherniavsky. 2014. Maliciously Circuit-Private FHE. In Advances in Cryptology - CRYPTO 2014. 536553. https://doi.org/10.1007/978-3-662-44371-2_30

[57] Rafael Pass. 2003. Simulation in Quasi-Polynomial Time, and Its Application to Protocol Composition. In Advances in Cryptology - EUROCRYPT 2003. 160-176. https://doi.org/10.1007/3-540-39200-9_10

[58] Amit Sahai and Salil P. Vadhan. 1997. A Complete Promise Problem for Statistical Zero-Knowledge. In 38th Annual Symposium on Foundations of Computer Science, FOCS '97, Miami Beach, Florida, USA, October 19-22, 1997. 448-457. https://doi. org/10.1109/SFCS.1997.646133

[59] Daniel Wichs and Giorgos Zirdelis. 2017. Obfuscating Compute-and-Compare Programs under LWE. In 58th IEEE Annual Symposium on Foundations of Computer Science, FOCS 2017, Berkeley, CA, USA, October 15-17, 2017. 600-611. https: //doi.org/10.1109/FOCS.2017.61 